\title{
A proposed grading system for standardizing tumor consistency of intracranial meningiomas
}

\author{
Gabriel Zada, M.D., Parham Yashar, M.D., Aaron Robison, M.D., Jesse Winer, M.D., \\ Alexander Khalessi, M.D., M.S., William J. Mack, M.D., \\ and Steven L. Giannotta, M.D.
}

Department of Neurosurgery, Keck School of Medicine of USC, University of Southern California, Los Angeles, California

\begin{abstract}
Object. Tumor consistency plays an important and underrecognized role in the surgeon's ability to resect meningiomas, especially with evolving trends toward minimally invasive and keyhole surgical approaches. Aside from descriptors such as "hard" or "soft," no objective criteria exist for grading, studying, and conveying the consistency of meningiomas.

Methods. The authors designed a practical 5-point scale for intraoperative grading of meningiomas based on the surgeon's ability to internally debulk the tumor and on the subsequent resistance to folding of the tumor capsule. Tumor consistency grades and features are as follows: 1) extremely soft tumor, internal debulking with suction only; 2) soft tumor, internal debulking mostly with suction, and remaining fibrous strands resected with easily folded capsule; 3) average consistency, tumor cannot be freely suctioned and requires mechanical debulking, and the capsule then folds with relative ease; 4) firm tumor, high degree of mechanical debulking required, and capsule remains difficult to fold; and 5) extremely firm, calcified tumor, approaches density of bone, and capsule does not fold. Additional grading categories included tumor heterogeneity (with minimum and maximum consistency scores) and a 3-point vascularity score. This grading system was prospectively assessed in 50 consecutive patients undergoing craniotomy for meningioma resection by 2 surgeons in an independent fashion. Grading scores were subjected to a linear weighted kappa analysis for interuser reliability.

Results. Fifty patients (100 scores) were included in the analysis. The mean maximal tumor diameter was 4.3 $\mathrm{cm}$. The distribution of overall tumor consistency scores was as follows: Grade 1,4\%; Grade 2, 9\%; Grade 3, 43\%; Grade 4, 44\%; and Grade 5, $0 \%$. Regions of Grade 5 consistency were reported only focally in $14 \%$ of heterogeneous tumors. Tumors were designated as homogeneous in $68 \%$ and heterogeneous in $32 \%$ of grades. The kappa analysis score for overall tumor consistency grade was 0.87 (SE 0.06, 95\% CI 0.76-0.99), with 90\% user agreement. Kappa analysis scores for minimum and maximum grades of tumor regions were 0.69 (agreement $72 \%$ ) and 0.75 (agreement 78\%), respectively. The kappa analysis score for tumor vascularity grading was 0.56 (agreement $76 \%$ ). Overall consistency did not correlate with patient age, tumor location, or tumor size. A higher tumor vascularity grade was associated with a larger tumor diameter $(\mathrm{p}=0.045)$ and with skull base location $(\mathrm{p}=0.02)$.

Conclusions. The proposed grading system provides a reliable, practical, and objective assessment of meningioma consistency and facilitates communication among providers. This system also accounts for heterogeneity in tumor consistency. With the proposed scale, meningioma consistency can be standardized as groundwork for future studies relating to surgical outcomes, predictability of consistency and vascularity using neuroimaging techniques, and effectiveness of various surgical instruments.
\end{abstract}

(http://thejns.org/doi/abs/10.3171/2013.8.FOCUS13274)

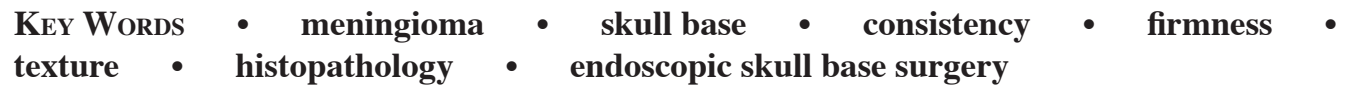

$\mathrm{O}$ VER the past decade, a great deal of interest has been generated in the resection of extraaxial skull base tumors via minimally invasive, endoscopic, and keyhole surgical approaches. Open surgical approaches for skull base meningiomas of the anterior and middle skull base, which still define the gold-standard intervention for many of these lesions, have been challenged by more recent series of endoscopic transnasal and keyhole approaches. Minimally invasive approaches have in turn been galvanized by advancements in endoscopic technology, instrumentation, and the development of novel techniques for skull base reconstruction and CSF leak repair. As neurosurgical approaches to meningiomas and other cerebral tumors continue to evolve in terms of minimal invasiveness, the selection of patients for minimally invasive versus traditional open surgical approaches is likely to become even more critical in optimizing patient outcomes. A priori knowledge of key tumor characteristics (including pathology, vascularity, invasiveness, and so on) acquired using advanced neuroimaging modalities is likely to contribute greatly to this patient selection process. 
One key characteristic of skull base tumors that remains rather elusive with regard to preoperative assessment, yet greatly affects the ease of the operation, is tumor consistency (also referred to as texture or firmness). In previous attempts to predict meningioma consistency based on neuroimaging studies, investigators have generally referred to the meningiomas as either "hard" or "soft" and have often reported this information in a retrospective fashion derived from operative notes. As neurosurgeons are increasingly having to decide between minimally invasive and open craniotomy approaches, tumor consistency is likely to become a more important factor in determining the operative procedure as well as any intraoperative or preoperative adjunctive measures. Furthermore, as newer and more advanced neuroimaging modalities provide improved means of assessing tumor consistency, a more detailed and objective tumor consistency scoring system would greatly improve our ability to analyze and convey surgical parameters relating to tumor consistency in a standardized fashion. Finally, the ability to objectively assess surgical tools for tumor resection may be improved by the application of a standardized grading system for tumor consistency.

For these reasons, the authors aimed to establish and test the validity of an objective and practical grading system for meningioma consistency, which could more accurately account for the continuous, rather than binary, spectrum of meningioma texture, as well as accounting for regions of intratumoral heterogeneity with respect to consistency. We created a practical 5-point grading system to quantify the consistency of intracranial meningiomas based on the ease of internal debulking and ability to fold in the tumor capsule following debulking. We subsequently assessed the validity of this 5-point grading system through the independent grading of each tumor by 2 neurosurgeons who were involved with the resection and who did not discuss the tumor grading during the operation.

\section{Methods}

\section{Consistency Score Development}

A 5-point grading scale was developed to characterize the consistency encountered during the resection of meningiomas (Table 1). The authors aimed to design a practical and objective scale, based on the surgeon's ease and ability to internally debulk the tumor and subsequently collapse the capsule of the meningioma, and the tools used to do so. Grade 1 meningiomas, those whose consistency is the softest subset of meningiomas, were defined as those amenable to internal debulking utilizing suction alone as a primary instrument. Grade 2 meningiomas are also relatively soft tumors that can be partially debulked via suction, yet subsequently retain an interspersing fibrous stroma that requires additional resection, along with an easily folded capsule. Grade 3 meningiomas are those with average consistency that cannot be freely suctioned and require some degree of mechanical debulking (that is, ultrasonic aspiration, piecemeal debulking, or cutting-aspiration device), with a capsule that is easily collapsed following internal debulking. Grade 4 meningiomas are firm tumors for which internal debulking is a challenge, often mandating sharp resection, loop cautery, ultrasonic aspiration, or mechanical fragmentation at a higher setting; in Grade 4 tumors, folding of the fibrous tumor capsule poses a challenge to the surgeon despite internal debulking. Finally, Grade 5 meningiomas are extremely fibrous or calcified lesions, with a consistency approaching bone, in which the paradigm of internal debulking and capsule folding is typically unsuccessful. When using the consistency score, tumors are graded based on the ease of internal debulking and stiffness of the tumor capsule, and they can therefore can be scored based on any combination of suction and/or mechanical fragmentation used.

To accommodate for potential heterogeneity in the consistency of meningiomas, tumors were secondarily categorized as homogeneous or heterogeneous and were subsequently graded with a minimum and maximum consistency corresponding to the softest and firmest parts of the tumor, according to the same grading scheme posed above. Finally, a 3-point grading system was used to characterize the vascularity of the tumor parenchyma (avascular, average, or vascular).

\section{Clinical Application of Consistency Grading Scale}

According to the linear weighted kappa model for interuser variability, the study was designed to have 50 patients, per the formula $2 \kappa^{2}$ for a 2 -rater assessment, where $\kappa$ is the number of categories $(\kappa=5) \cdot{ }^{3-5}$ Linear weighted kappa analysis was selected based on an ordinal grading scale with no weight differences between categories. After obtaining institutional review board approval, we began using the consistency grading scale in a prospective fashion, applying it in 50 consecutive cranial operations for meningiomas (49 cases involving open craniotomy and 1 case involving an endoscopic endonasal approach for olfactory groove meningioma). For the purposes of this study, patients with smaller tumors that were resected in an en bloc fashion (without requiring internal debulking) and patients undergoing preoperative embolization were excluded. Each grader was an attending neurosurgeon or chief resident who had enough experience with meningioma operations to discriminate among varying tumor consistencies, and all participants were given brief instruction on how to use the scale and complete the grading forms prior to the operation. Immediately following each operation, tumors were graded, in an independent fashion and by following a questionnaire, by 2 surgeons who performed the operation but did not communicate regarding the grading score.

\section{Statistical Analysis}

Following the grading of 50 tumors, data were analyzed according to a standard linear kappa statistical analysis for interuser variability. A kappa score of greater than 0.8 signifies an excellent test with almost perfect agreement, and a kappa score of $0.6-0.8$ signifies a good test with substantial interuser reliability (Table 2). ${ }^{10}$ Proportional data among categories were compared using 


\section{Grading system for intracranial meningioma consistency}

TABLE 1: Meningioma consistency grading system

\begin{tabular}{|c|c|c|c|}
\hline $\begin{array}{l}\text { Consistency } \\
\text { Score }\end{array}$ & General Description & Capsule Characteristics & $\begin{array}{l}\text { Exemplary Instrument(s) Used } \\
\text { for Internal Debulking }\end{array}$ \\
\hline 1 & extremely soft tumor & $\begin{array}{l}\text { capsule easily folded or no } \\
\text { capsule }\end{array}$ & suction \\
\hline 2 & $\begin{array}{l}\text { soft tumor; bulk of tumor freely suctioned; fibrous } \\
\text { stroma remains }\end{array}$ & $\begin{array}{l}\text { capsule easily folded following } \\
\text { partial internal debulking }\end{array}$ & $\begin{array}{l}\text { majority done w/ suction; fibrous stroma resected } \\
\text { w/ capsule }\end{array}$ \\
\hline 3 & $\begin{array}{l}\text { average consistency; cannot be freely suctioned; } \\
\text { requires some degree of mechanical debulking }\end{array}$ & $\begin{array}{l}\text { capsule folds following suffi- } \\
\text { cient debulking }\end{array}$ & $\begin{array}{l}\text { piecemeal resection; ultrasonic aspiration or } \\
\text { other mechanical debulking device, often at } \\
\text { low setting; sharp dissection }\end{array}$ \\
\hline 4 & firm tumor; requires mechanical debulking & $\begin{array}{l}\text { firm capsule; difficult to collapse } \\
\text { despite tumor debulking }\end{array}$ & $\begin{array}{l}\text { piecemeal resection, ultrasonic aspiration, loop } \\
\text { cautery, or other mechanical debulking, often } \\
\text { at high setting; sharp resection or loop cautery }\end{array}$ \\
\hline 5 & $\begin{array}{l}\text { extremely firm \&/or calcified tumor; may approach } \\
\text { density of bone; often requires en bloc resection }\end{array}$ & $\begin{array}{l}\text { rigid capsule that does not fold } \\
\text { or collapse }\end{array}$ & $\begin{array}{l}\text { difficult to debulk even w/ ultrasonic aspiration, } \\
\text { cautery loop, or mechanical/sharp dissection }\end{array}$ \\
\hline
\end{tabular}

Fisher's exact test. Tumor size was analyzed as categorical data (diameter $<3 \mathrm{~cm}, 3-5 \mathrm{~cm}$, or $>5 \mathrm{~cm}$ ), as was tumor location (convexity vs skull base). Statistical significance was defined as $\mathrm{p}<0.05$. All statistical analyses were performed using SPSS (SPSS Inc.).

\section{Results}

\section{Patient Demographics and Tumor Location}

Fifty intracranial meningiomas in 50 patients were graded by 2 independent reviewers (100 scores total). The mean patient age was 52 years. There were 11 men and 39 women. The mean maximal tumor diameter $( \pm$ SD) was $4.3 \pm 1.5 \mathrm{~cm}$. Tumors could be broadly categorized as follows: skull base (52\%), convexity/parafalcine (44\%), and intraventricular (4\%). The specific distributions of tumor location are shown in Table 3 . A prior resection had been attempted in 2 patients (4\%). In 2 patients ( 1 who had a prior resection), radiation therapy had previously been performed. The distribution of scores for overall, minimum, and maximum grading is depicted in Fig. 1.

\section{Interuser Reliability of Grading System for Consistency}

The overall tumor consistency grade was perfectly correlated in 45 of the 50 cases (a 90\% agreement). The kappa score for overall consistency grading was 0.87 (SE $0.06,95 \%$ CI 0.76-0.99) (Table 4). The designation of

TABLE 2: Magnitudes of interuser agreement stratified by kappa score* $^{*}$

\begin{tabular}{cl}
\hline Kappa Score Range & Magnitude of Agreement \\
\hline$<0$ & no agreement \\
$0-0.20$ & slight agreement \\
$0.21-0.40$ & fair agreement \\
$0.41-0.60$ & moderate agreement \\
$0.61-0.80$ & substantial agreement \\
$0.81-1$ & almost perfect agreement \\
\hline
\end{tabular}

* Based on the study by Landis and Koch, 1977. "homogeneity versus heterogeneity" of tumor consistency was correlated in 42 of 50 patients (an 84\% agreement). The kappa score for homogeneity versus heterogeneity was 0.64 (SE 0.12, 95\% CI 0.41-0.86). Rater agreement was observed in the minimum tumor consistency score in $74 \%$ of tumors, with a kappa score of 0.69 (SE 0.08, 95\% CI 0.52-0.85). Rater agreement was observed in the maximum tumor consistency score in $78 \%$ of tumors, with a kappa score of 0.75 (SE 0.07, 95\% CI 0.61-0.90). The tumor vascularity grade (avascular, average, or vascular) was in agreement in $76 \%$ of patients, with a kappa score of 0.56 (SE 0.11, 95\% CI 0.34-0.78).

There was no statistically significant correlation between patient age; tumor diameter; overall, minimum, and maximum consistency; or tumor heterogeneity. A higher graded tumor vascularity, however, was associated

TABLE 3: Locations of 50 meningiomas included in current study

\begin{tabular}{lcc}
\hline \multicolumn{1}{c}{ Category \& Location } & No. of Lesions & Percentage \\
\hline convexity/parafalcine & & \\
parafalcine & 8 & 16 \\
convexity & 14 & 28 \\
total & 22 & 44 \\
skull base & & \\
sphenoid wing & 5 & 10 \\
olfactory groove & 5 & 10 \\
tentorial & 4 & 8 \\
tuberculum sella & 3 & 6 \\
cerebellopontine angle & 3 & 6 \\
clinoidal & 2 & 4 \\
middle fossa & 1 & 2 \\
petroclival & 1 & 2 \\
planum sphenoidale & 1 & 2 \\
total & 26 & 52 \\
intraventricular & & 4 \\
$\quad$ atrium of lateral ventricle & 2 & 100 \\
overall total & 50 & \\
\hline
\end{tabular}




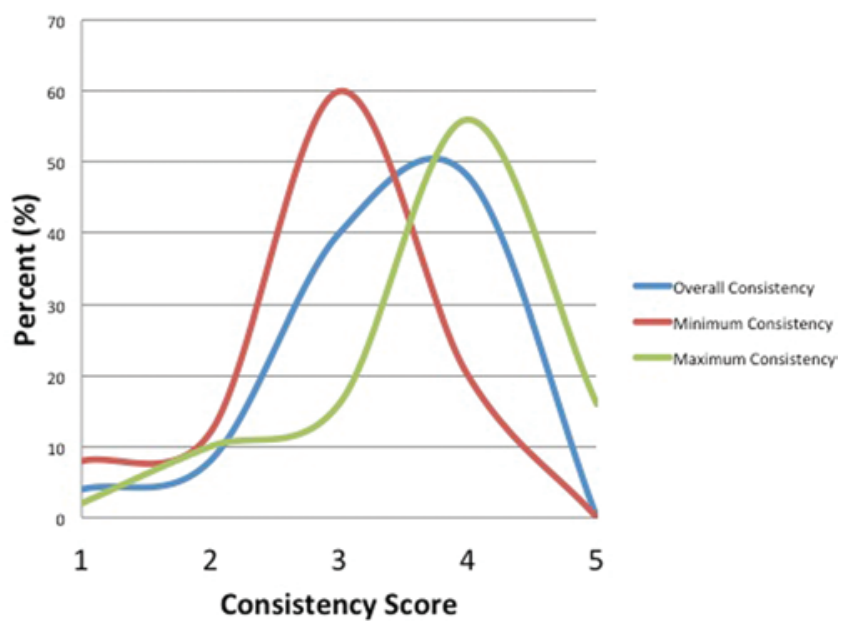

FIG. 1. Graph demonstrating the distribution of consistency scores (by percentage) for 50 graded meningiomas. The three lines represent the overall (blue), minimum (red), and maximum (green) consistency scores. Each score represents the average score from both graders.

with a larger tumor diameter $(>5 \mathrm{~cm})(\mathrm{p}=0.045$, Fisher's exact test) and with a skull base location ( $\mathrm{p}=0.020$, Fisher's exact test).

\section{Discussion}

Some of the key factors that affect the decision-making process for the surgical approach and the ease with which intracranial meningiomas can be resected are inherent tumor biology, size, growth patterns, invasion of surrounding neurovascular structures, vascular origins, and previous intervention(s).$^{18}$ Many authors have reported that firm consistency, or texture, is also a major limitation in the surgeon's ability to achieve optimal or complete resection of intracranial meningiomas. . $^{911,14,16,18}$ The grading system proposed in the current study provides a practical and easy-to-use method for the objective assessment of tumor consistency in intracranial meningiomas. It was created in response to the existing variability and subjectivity of prior studies that have attempted to characterize, predict, and convey meningioma consistency, often in a retrospective fashion. Furthermore, over the past few decades the inherent property of tumor consistency has become an increasingly important variable of meningioma surgery, as evolving minimally invasive options exist for removing a variety of intracranial skull base tumors. The ability to accurately predict the consistency of a meningi- oma in an a priori fashion based on neuroimaging studies may in part dictate the surgical approach one selects and provide more information regarding the requirement for a staged resection, expected subtotal resection, or additional challenges related to consistency and/or vascularity.

The grading system used in our study demonstrated a very high degree of agreement (90\%), with a kappa score of 0.87 , and also reliably accounted for the heterogeneity and vascularity of the tumor, albeit to a lesser degree. Finally, the practicality of the proposed grading system relies on standard microsurgical techniques typically used to resect meningiomas-namely, internal debulking of the tumor and subsequent folding of the tumor capsule. The flexibility in the grading of meningioma consistency based on various surgical instruments used for internal debulking also facilitates comparison of surgical instruments and newer surgical tools designed for tumor fragmentation and mechanical disruption. In the future, this scoring system may be used to objectively and practically assess current methods for tumor debulking and may help to accommodate newer tools that become available over the next several decades. Conversely, one limitation of the current system is that it may not be valid or generalizable to smaller meningiomas resected in an en bloc fashion or those that had undergone preoperative embolization, which were excluded from the present study.

In the future, the surgeon's ability to identify extremes in consistency in an a priori fashion-namely, Grade 1 and 2 versus Grade 4 and 5 tumors-is likely to help yield a great deal of information as to how a resection may proceed and help to guide the selection of a surgical approach. For example, in a meningioma known to have a preoperative Grade 1 consistency based on neuroimaging, a minimally invasive approach in which suction and minimal curettage would be sufficient for resection could be reliably planned. At the opposite extreme, a Grade 5 meningioma is more likely to require a more substantial open exposure that involves bony removal and possibly brain retraction, knowing that internal debulking strategies may be rather limited. Although in the current series no meningiomas received an overall consistency grade of $5,14 \%$ of the tumors did have focal regions of Grade 5 consistency. Both our experience and prior studies support the requirement for maintaining such a category. ${ }^{8,13}$ Another interesting question is whether the consistency of a tumor has any effect on its response or resistance to radiation-based treatments such as Gamma Knife surgery. This question may be more objectively assessed in the future based on the current grading system.

TABLE 4: Statistics for graded variables according to proposed system in 50 meningiomas graded by 2 users

\begin{tabular}{lcccccccc}
\hline \multicolumn{1}{c}{ Grading Variable } & $\begin{array}{c}\text { No. of } \\
\text { Categories }\end{array}$ & $\begin{array}{c}\% \\
\text { Agreement }\end{array}$ & $\begin{array}{c}95 \% \mathrm{Cl} \text { for } \\
\text { Agreement (\%) }\end{array}$ & $\begin{array}{c}\text { Kappa } \\
\text { Score }\end{array}$ & $\begin{array}{c}\text { Max Possible } \\
\text { Kappa }\end{array}$ & $\begin{array}{c}\text { Standard } \\
\text { Error }\end{array}$ & $\begin{array}{c}95 \% \text { Cl for } \\
\text { Kappa Score }\end{array}$ & $\begin{array}{c}\text { Proportion of Max } \\
\text { Possible Kappa (\%) }\end{array}$ \\
\hline overall consistency & 5 & 90 & $77-96$ & 0.87 & 0.92 & 0.056 & $0.76-0.99$ & 95 \\
heterogeneity & 2 & 84 & $70-92$ & 0.67 & 0.82 & 0.115 & $0.41-0.86$ & 78 \\
minimum consistency & 5 & 74 & $59-84$ & 0.69 & 0.91 & 0.083 & $0.52-0.85$ & 75 \\
max consistency & 5 & 78 & $64-88$ & 0.75 & 0.92 & 0.074 & $0.61-0.90$ & 82 \\
vascularity & 3 & 76 & $62-86$ & 0.57 & 0.78 & 0.112 & $0.38-0.78$ & 72 \\
\hline
\end{tabular}




\section{Grading system for intracranial meningioma consistency}

It is believed that the consistency of meningiomas is in part dictated by the content of collagen and water, but to date no sustained or universally accepted correlation with histopathological subtype has ever been substantiated. Over the past 2 decades, numerous studies have attempted to predict the consistency of cranial meningiomas by using a variety of advanced MRI techniques, ranging from T2-weighted to diffusion-tensor imaging to MR elastography sequences, mainly with limited success. ${ }^{1,2,6,7,15}$ A prior study by Chen et al., from our institution, did show a correlation between soft tumor consistency and hyperintensity on T2-weighted MRI, ${ }^{2}$ as have some additional studies. ${ }^{6,9,12,15,19}$ Two prior studies have demonstrated an association between hard tumors and the fibroblastic meningioma subtype.,7 Other studies, however, have found no relationship between imaging findings and consistency ${ }^{17}$ or between histology and consistency. ${ }^{17}$ The greatest success, to date, in demonstrating a consistency-imaging relationship has been a study utilizing diffusion-tensor imaging by Kashimura et al., who in 2007 reported an improved ability to predict tumor consistency as "hard" or "soft" in 29 patients with meningiomas.7 Nearly all previous studies have referred to meningiomas as "hard" or "soft," however, which does not account for the more continuous spectrum and heterogeneity frequently observed in the tumor's consistency and does not represent a standardized scale that has been validated prior to its use.

The major aim of the current study was to provide a practical platform for neurosurgeons, neuroradiologists, neuropathologists, and other practitioners to objectively and reliably convey meningioma characteristics relating to consistency and vascularity, as new imaging modalities and minimally invasive surgical techniques continue to evolve rapidly. Our study does have certain limitations, however, in that the interuser agreement and kappa scores of some aspects of the scoring system-namely, the heterogeneous components and tumor vascularity-were not as reliable as those for the overall consistency score. Although agreement was noted in the $70 \%-80 \%$ range, and the kappa score was still in the $0.61-0.80$ range for these characteristics (indicating a substantially reliable test), they are nevertheless not perfectly reliable grading systems and are still subject to interuser disagreement. Even the lower margins of the $95 \%$ confidence intervals for these scores, however, indicate a moderately reliable test for these categories. The main variable that is useful for grading is the overall consistency score, with a kappa score of 0.87 and $90 \%$ interuser agreement. Furthermore, at this time the proposed scale does not hold clinical validity or association with any established outcome parameters for meningiomas. Further use of the grading scale may or may not demonstrate such an association in the future. Finally, the purpose of this study was not to definitively draw associations between tumor consistency and neuroimaging findings or histopathological subtype. The aim of this study was only to validate the objectiveness of this scoring system, with further results drawing correlations to imaging and pathology findings to be analyzed once a sufficient number of tumors have been graded according to this scoring system.

\section{Conclusions}

The grading system developed and tested in the current study represents a simple, practical, and objective method for assessing the consistency of intracranial meningiomas and was noted to have an extremely high degree of interuser reliability, especially for overall consistency score, which was the main variable assessed. The application of such a grading score may standardize the communication of meningioma texture among practitioners and improve the objectivity and comparison of future research studies in terms of the predictability of intraoperative consistency using advanced neuroimaging techniques as well as effectiveness of various instruments for tumor debulking.

\section{Acknowledgment}

We would like to thank Anush Arakelyan, M.P.H., for her help with statistical analysis.

\section{Disclosure}

The authors report no conflict of interest concerning the materials or methods used in this study or the findings specified in this paper.

Author contributions to the study and manuscript preparation include the following. Conception and design: Zada, Giannotta. Acquisition of data: Zada, Yashar, Winer, Robison, Khalessi. Analysis and interpretation of data: Zada, Winer, Robison, Khalessi, Mack. Drafting the article: Zada. Critically revising the article: Zada, Yashar, Winer, Mack, Giannotta. Reviewed submitted version of manuscript: all authors. Approved the final version of the manuscript on behalf of all authors: Zada. Statistical analysis: Zada, Khalessi, Mack. Administrative/technical/material support: Yashar, Winer, Robison. Study supervision: Zada, Giannotta.

\section{References}

1. Carpeggiani P, Crisi G, Trevisan C: MRI of intracranial meningiomas: correlations with histology and physical consistency. Neuroradiology 35:532-536, 1993

2. Chen TC, Zee CS, Miller CA, Weiss MH, Tang G, Chin L, et al: Magnetic resonance imaging and pathological correlates of meningiomas. Neurosurgery 31:1015-1022, 1992

3. Cicchetti DV: Assessing inter-rater reliability for rating scales: resolving some basic issues. Br J Psychiatry 129:452-456, 1976

4. Cicchetti DV: Testing the normal approximation and minimal sample size requirements of weighted kappa when the number of categories is large. Appl Psychol Meas 5:101-104, 1981

5. Cicchetti DV, Fleiss JL: Comparison of the null distribution of weighted Kappa and the C ordinal statistic. Appl Psychol Meas 1:195-201, 1977

6. Hoover JM, Morris JM, Meyer FB: Use of preoperative magnetic resonance imaging $\mathrm{T} 1$ and $\mathrm{T} 2$ sequences to determine intraoperative meningioma consistency. Surg Neurol Int 2: 142,2011

7. Kashimura H, Inoue T, Ogasawara K, Arai H, Otawara Y, Kanbara Y, et al: Prediction of meningioma consistency using fractional anisotropy value measured by magnetic resonance imaging. J Neurosurg 107:784-787, 2007

8. Kato K, Chernov M, Urino T, Kasuya H, Kubo O, Iseki H, et al: Ossified frontosphenoorbital meningioma en plaque, mimicking extensive hyperostosis. Minim Invasive Neurosurg 51:237-239, 2008

9. Kim TW, Jung S, Jung TY, Kim IY, Kang SS, Kim SH: Prog- 


\section{G. Zada et al.}

nostic factors of postoperative visual outcomes in tuberculum sellae meningioma. Br J Neurosurg 22:231-234, 2008

10. Landis JR, Koch GG: An application of hierarchical kappatype statistics in the assessment of majority agreement among multiple observers. Biometrics 33:363-374, 1977

11. Little KM, Friedman AH, Sampson JH, Wanibuchi M, Fukushima T: Surgical management of petroclival meningiomas: defining resection goals based on risk of neurological morbidity and tumor recurrence rates in 137 patients. Neurosurgery 56:546-559, 2005

12. Maiuri F, Iaconetta G, de Divitiis O, Cirillo S, Di Salle F, De Caro ML: Intracranial meningiomas: correlations between MR imaging and histology. Eur J Radiol 31:69-75, 1999

13. Patankar T, Prasad S, Goel A: Sphenoid wing meningiomaan unusual cause of duro-optic calcification. J Postgrad Med 43:48-49, 1997

14. Sekhar LN, Jannetta PJ, Burkhart LE, Janosky JE: Meningiomas involving the clivus: a six-year experience with 41 patients. Neurosurgery 27:764-781, 1990

15. Suzuki Y, Sugimoto T, Shibuya M, Sugita K, Patel SJ: Meningiomas: correlation between MRI characteristics and operative findings including consistency. Acta Neurochir (Wien) 129:39-46, 1994
16. Tahara A, de Santana PA Jr, Calfat Maldaun MV, Panagopoulos AT, da Silva AN, Zicarelli CA, et al: Petroclival meningiomas: surgical management and common complications. J Clin Neurosci 16:655-659, 2009

17. Yamaguchi N, Kawase T, Sagoh M, Ohira T, Shiga H, Toya S: Prediction of consistency of meningiomas with preoperative magnetic resonance imaging. Surg Neurol 48:579-583, 1997

18. Zada G, Du R, Laws ER Jr: Defining the "edge of the envelope": patient selection in treating complex sellar-based neoplasms via transsphenoidal versus open craniotomy. Clinical article. J Neurosurg 114:286-300, 2011

19. Zee CS, Chin T, Segall HD, Destian S, Ahmadi J: Magnetic resonance imaging of meningiomas. Semin Ultrasound CT MR 13:154-169, 1992

Manuscript submitted June 27, 2013.

Accepted August 1, 2013.

Please include this information when citing this paper: DOI: 10.3171/2013.8.FOCUS13274.

Address correspondence to: Gabriel Zada, M.D., Department of Neurosurgery, University of Southern California, 1200 N. State St., Ste. 5046, Los Angeles, CA 90089. email: gzada@usc.edu. 